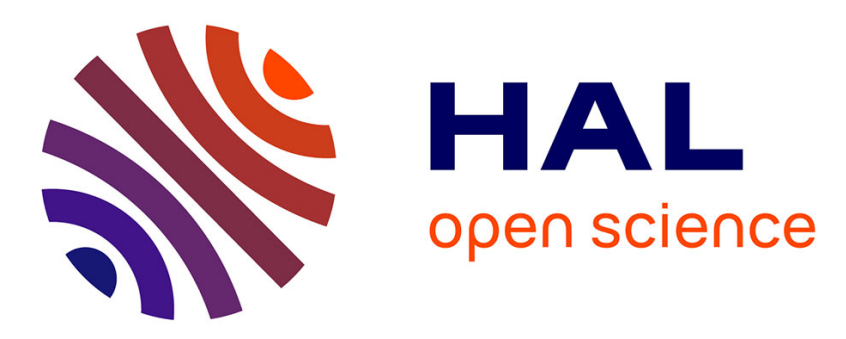

\title{
Imaging Biomarker Ontology (IBO): A Biomedical Ontology to Annotate and Share Imaging Biomarker Data
}

\author{
Emna Amdouni, Bernard Gibaud
}

\section{- To cite this version:}

Emna Amdouni, Bernard Gibaud. Imaging Biomarker Ontology (IBO): A Biomedical Ontology to Annotate and Share Imaging Biomarker Data. Journal on Data Semantics, 2018, 7 (4), pp.223. 10.1007/s13740-018-0093-3 . hal-01887970

\section{HAL Id: hal-01887970 \\ https://hal.science/hal-01887970}

Submitted on 4 Oct 2018

HAL is a multi-disciplinary open access archive for the deposit and dissemination of scientific research documents, whether they are published or not. The documents may come from teaching and research institutions in France or abroad, or from public or private research centers.
L'archive ouverte pluridisciplinaire HAL, est destinée au dépôt et à la diffusion de documents scientifiques de niveau recherche, publiés ou non, émanant des établissements d'enseignement et de recherche français ou étrangers, des laboratoires publics ou privés. 


\title{
Imaging Biomarker Ontology (IBO): A Biomedical Ontology to Annotate and Share Imaging Biomarker Data
}

\author{
Emna Amdouni · Bernard Gibaud
}

Received: 22 July 2017 / Accepted: 31 August 2018

\begin{abstract}
Imaging biomarkers refer to radiological measurements that characterize biological processes of imaged subjects and help clinicians particularly in the assessment of therapeutic responses and the early prediction of pathologies. Several imaging features (size of a lesion, volume of a tumor, blood perfusion in a specific anatomical region, anisotropic water diffusion in a particular tissue region, etc.) are quantified and reported in the clinical practice. The growth of the number of research studies addressing imaging biomarkers and the increasing use of these measurements in the radiological routine necessitates the use of semantic research tools. The use of semantic technologies will enable to efficiently retrieve imaging related data and to enhance the interoperability in the biomedical field.

While many efforts have been conducted regarding the definition of a standardized vocabulary to support the sharing of the imaging biomarker knowledge, the definition of the term "imaging biomarker" stills inconsistent. In this paper, we introduce our motivation for semantically describing this concept and we outline shortcomings of the state-of-the art methods. Here, we propose a semantic representation of the imaging biomarker concept that is based on the articulation of its three main semantic axes, namely the measured quality, the measurement tool and the decision tool. The developed ontology is called the Imaging Biomarker Ontology (IBO) and uses existing biomedical ontologies. A preliminary use case is studied to illus-
\end{abstract}

Emna Amdouni

Bcom Institute of Research and Technologies, Rennes, France LTSI Inserm 1099, Université de Rennes 1

E-mail: emnaamdouni89@gmail.com

Bernard Gibaud

Bcom Institute of Research and Technologies, Rennes, France LTSI Inserm 1099, Université de Rennes 1 trate the utility of IBO in annotating quantitative and qualitative imaging data from the TCGA (The Cancer Genome Atlas) collection.

Keywords knowledge representation · imaging biomarker · ontology development · biomedical ontologies

\section{Background}

Quantitative imaging (Kessler et al., 2015) refers to the extraction and use of numerical characteristics that are extracted from medical images. Its ultimate objective is to improve multi-scale understanding of pathologies at the anatomical, functional and molecular level through the use of advanced digital techniques (Jaffer and Weissleder, 2005). The National Institute of Health has defined the concept of imaging biomarker as "characteristics that are objectively measured and evaluated as an indicator of normal biological processes, pathogenic process or biological responses to a therapeutic intervention" (Wagner et al., 2007). For example, Response Evaluation Criteria in Solid Tumors (RECIST) (Eisenhauer et al., 2009) enable clinicians to assess tumor evolution via the measurement of the tumor size from Computed Tomography (CT) images.

\subsection{The importance of the Imaging Biomarker Concept}

The importance of biomarkers in general is widely acknowledged in the scientific literature, due to their potential role in future clinical practice (Buckler et al., 2011; ESR, 2010), but also due to the critical role biomarkers play in the development of new drugs, e.g., as surrogate endpoints in clinical trials (Kelloff 
et al., 2004). Notably, imaging biomarkers allow testing new drugs at an early non-symptomatic stage of pathology, potentially leading to significant reduction in costs (Abramson and Yankeelov, 2014; O'Connor et al., 2008). This perspective of wide-scale use and reuse of imaging biomarkers in medical research and clinical practice makes it very important to be able to share information about biomarkers, e.g., what they actually measure and their degree of validation for specific intended uses (e.g., link with clinical outcome).

\subsection{Challenges}

It becomes important to be able to share information relative to imaging biomarkers to ensure understanding, reuse and integration of their meta-data and support clinical research studies (Asslaber and Zatloukal, 2007; Hewitt and Watson, 2013). However, this concept is not easy to define because it involves multiple notions such as the measured biological quality, the measurement process and the decision making tool. Till now, the way the term "imaging biomarker" is used in literature is somewhat confusing. In 2010, the Institute of Medicine stressed the need to define a consistent and precise way the vocabulary related to biomarkers (Ball et al., 2010).

Recent works from different communities (imaging research, clinical radiology, genetics, pharmaceutical industry, knowledge management, etc.) showed interest in consistently representing imaging biomarkers (Ofoghi et al., 2014). Actually, none of the existing works in the literature has succeeded in defining a vocabulary that covers this domain in a coherent way. In some works (Ceusters and Smith, 2015), this term refers to some substance that can be assessed, and in others (RSNA, 2007), it refers to a measurement method which ensures a reproducible and precise measurement of imaging biomarker values. In other cases (ESR, 2010), it is considered as a decision making tool used to assess the progression of some pathology (e.g., cancer, Alzheimer's disease and cardiovascular diseases) or the response to some therapeutic intervention. For the reasons cited above and given the large scale use of the imaging biomarker concept, we propose here to define in a coherent way the vocabulary associated with it. $\mathrm{f}$

\subsection{Objective}

The objective of this work is to define explicitly, by means of an ontology, the vocabulary pertaining to imaging biomarkers. Our proposed ontology is entitled Imaging Biomarker Ontology (IBO), and it is based on existing biomedical ontologies. Ontologies are widely acknowledged as a means to specify explicitly the meaning of concepts in a domain of interest, and to facilitate consistent sharing of data and knowledge pertaining to them.

In our context, ontologies can help addressing the major challenges mentioned above, by providing: (1) a standard vocabulary to describe imaging biomarker information, covering and articulating the various meanings attached to this term in the medical imaging and image processing communities, and (2) a formal language to reason about this information. The latter will be more and more needed in the future in the context of the development of decision support systems that will be involved in medical decision processes, such as choosing the diagnostic procedure that is best suited to the patient case. Such choice will involve some reasoning aiming at determining which quantitative imaging biomarkers data are best appropriate to diagnose the patient, and then choosing the relevant image acquisition protocol and organizing the acquisition and processing of the images in such a way to deliver the imaging biomarkers. Once available, these biomarkers can be used, together with other medical data relevant to the patient, to make actual medical decisions about therapy and patient management.

Our work addresses the specific goal of delivering a core ontology, supposed to be valid across the whole domain of medical imaging, but still relatively abstract. Once validated this core ontology will be further extended to cover the specific needs related to the various imaging modalities, medical specialties and diseases. In this paper, we will illustrate how IBO can be used to annotate imaging biomarkers results and answer to some competency questions as:

- Q1: retrieve all imaging biomarker data about (patient, images, measurements, etc.) in studies of patients with a specific disease (GBM, Alzheimer, etc.).

- Q2: find all regions of interest (ROI) of images and retrieve their associated values.

- Q3: describe how the imaging biomarker values were obtained; what processes were executed?

- Q4: find all scalar measurements associated to a given imaging study.

- etc.

This paper is organized as follows: sect 2 presents a survey of existing works related to the representation of imaging biomarkers and it outlines their limitations. In sect 3 , we define the scope and methodology of our work and the existing ontologies which have been reused. In Sect 4 entitled development of the Imaging Biomarker Ontology, the structure and the most salient aspects of 
our ontology are detailed. Section 5 gives some illustrative examples to show how we can annotate imaging features with the IBO ontology. Finally, the advantages and limits of our work are discussed in Sect 6 .

\section{Related Works}

\subsection{DICOM Structured Reports Format}

The DICOM standard (Digital Imaging and Communications in Medicine) specifies a data structure for SR (Structured Reports) (Clunie, 2000) as a set of rules constraining their organization and a vocabulary (coded concepts associated to their meanings) covering the domain of imaging observations. Such DICOM SR objects allow representing a wide range of imaging observations, including measurements and qualitative assessments, (e.g., the presence or not of a mass, its dimension and its position), their relationships with image evidence and with inferred diagnosis.

Despite the wide use of the DICOM standard in clinical practice, DICOM SR has had limited success for exchanging imaging reports in clinical settings. In this survey (Bosmans et al., 2012), radiologists expressed that the use of DICOM SR is a time- and an energyconsuming task. Moreover, they mentioned that the use of SR templates had restricted their freedom in terms of expression given that they do not enable them to add free texts to enrich the contents of their reports. Most participants to this survey pointed out the necessity to find a better way to enter input data so that the implementation of SR does not affect productivity.

Only a limited number of imaging biomarkers can be represented using DICOM SR, yet, together with their associated provenance data. Clunie et al. Clunie (2007) have proposed extensions of (e.g., DICOM codes and SR templates) to facilitate encoding and exchange of oncology clinical trial results from this work. Moreover, DICOM has intrinsic limitations regarding querying; the absence of formal definitions makes the reasoning on DICOM data difficult. DICOM data should be complemented with data expressed in an ontological format to improve data querying capabilities. Until today, there is no DICOM ontology, although some research has focused on the question of the semantic representation of the DICOM standard, e.g., (Brunnbauer, 2013).

\subsection{Annotation and Image Markup Model}

The AIM (Annotation and Image Markup) model (Channin et al., 2010) was developed within the frame- work of the caBIG (Cancer Biomedical Informatics Grid). This information model was designed to support the representation of the radiological annotations that refer to measurements, texts, observations, graphic shapes delimiting regions of interest, etc. The AIM model is used to manage radiological annotations in the research context. AIM data can be stored in XML files and open source tools AIM (i.e., API Web Page ${ }^{1}$, ipad tool (Rubin et al., 2008)) exist to help developers in the serialization of AIM data into their application.

In summary, the AIM model has introduced the most relevant entities in image annotation, but its implementation lacks formal semantic because it is not based on ontologies. As a consequence, we can not represent complex entities or perform logic-based to infer new knowledge about the content of the image.

\subsection{Quantitative Imaging Biomarker Alliance}

In order to overcome the problems of standardization and to ensure the reproducibility of imaging biomarkers, the QIBA (Quantitative Imaging Biomarker Alliance) group of the RSNA (Radiological Society of North America) (Clunie, 2007) has defined standardized profiles to formalize the definition, generation and use of quantitative imaging biomarkers in clinical trials and clinical practice:

- The QIBA profile "Fluorodeoxyglucose (FDG)$\mathrm{PET} / \mathrm{CT}$ as an Imaging Biomarker Measuring Response to Cancer Therapy" indicates the technical specifications that are suitable for quantifying the absorption of the FDG tracer by tumor tissues. The FDG is a glucose analogue that enables the detection of the increase in the consumption of glucose by the tumor tissues (In cancerology, cancer cells capture FDG more than normal cells). Standardized approaches to the measurement of SUV (Standardized Uptake Value) and machine calibrations are described in the document.

- The QIBA profile "CT tumor change volume" provides standardized methods for measuring the volume of a lesion in a reproducible and repeatable manner to evaluate therapeutic response of patients. - etc.

The goal of this initiative is to "establish processes and profiles that will lead to acceptance of quantitative imaging biomarkers by the imaging community, clinical trial industry, and regulatory agencies as proof of biology, proof of changes in pathophysiology, and surrogate

1 https://web.stanford.edu/group/qil/cgibin/mediawiki/index.php/AIM-API 
endpoints for changes in the health status of patients." The aim of QIBA profiles is to standardize the production and use of imaging biomarker data by means of imaging protocols. Until now, these profiles are presented in free text format, which is certainly relevant for assisting clinical users in the definition of image acquisition and image processing protocols, but insufficient with regard to providing information models for implementing the interoperable repositories of imaging biomarker data that are needed in both medical facilities and for biomedical research.

Nevertheless, QIBA profiles can be of great help for defining important aspects that need to be considered to correctly cover the imaging biomarkers domain, especially the different steps that compose the measurement process and the modalities of their execution (used tools, imaging parameters, etc.).

\subsection{Quantitative Imaging Biomarker Ontology}

Buckler et al. have proposed the QIBO (Quantitative Imaging Biomarker Ontology) (Buckler et al., 2013) to improve the management of imaging biomarker data and to enable advanced research on image databases. This initiative is the first to use semantic web technologies to represent concepts related to quantitative imaging biomarkers. QIBO is a first attempt for untangling the various entities involved, especially biological subject, biological intervention, contrast agent, biological target, imaging instrument, algorithm, measurement, indicated biology and biomarker application. Nevertheless, this project, stalled and the model is far from being usable.

QIBO has three main limits. First, it does not reuse any foundational ontology, which is a major problem because the domain of imaging biomarker involves many complementary domains, for which ontologies exist but need to be consistently integrated. Second, QIBO does not reuse any existing ontology to cover the domain. Third, it lacks internal consistency, which is partly due to the lack of formal definitions of its entities and object properties.

For example, in terms of modeling, QIBO does not provide any formal axioms to relate the class QIBO:quantitative imaging biomarker to classes that describe provenance (biological quality, measurement method, etc.) of the measured value. Moreover, the classification of qualitative and quantitative imaging biomarkers should be revised given that it is not correct. For example, the class QIBO: shape parameter is defined as a QIBO:quantitative imaging biomarker whereas it describes a PATO: shape, which is defined as a qualitative quality.
2.5 Biomarker Retrieval and Knowledge Reasoning System

The BiomRKRS system (Biomarker Retrieval and Knowledge Reasoning System) (Ofoghi et al., 2014) is a similar project but with a broader scope (i.e., not limited to imaging biomarkers), with particular emphasis on providing a model for facilitating the interoperation of biomarkers' databases. In contrast with QIBO, BiomRKRS has reused existing relevant domain ontologies (e.g., Gene Ontology (Gene Ontology Consortium et al., 2008), Experimental Factor Ontology (Malone et al., 2010), QIBO) and terminologies (Systematized Nomenclature of Medicine - clinical terms, Logical Observation Identifier Names and Codes, ${ }^{2}$, International Classification of Diseases, ${ }^{3}$, etc.). However, the BiomRKRS ontology has not been made broadly available for reuse, yet.

2.6 Representation Based on the Ontology for General Medical Science (OGMS)

In this paper (Ceusters and Smith, 2015), Ceusters and Smith have used the OGMS ontology (Ontology for General Medical Science) (Scheuermann et al., 2009) to define the biomarker concept as an observable and evaluable characteristic, i.e. "a characteristic that is always identifiable in a process of observation and evaluation". Thus, they have defined the biomarker as an OGMS:bodily feature which subsumes three categories of biomarkers which are disjoint. The first category is the Material biomarker which refers to the anatomical structure OGMS: bodily component, the second category is the Quality biomarker which describes the quality associated with the observed anatomical structure (modeled as an OGMS:bodily quality), and the third category is the biomarker process which evaluates whether the process performed is normal or pathological (modeled as an OGMS: bodily process). Therefore, according to them the semiformal definition of the biomarker concept is as follows: Biomarker $=$ def. Material Biomarker, Quality Biomarker or Process Biomarker. The first limitation of this proposal is that it has defined biomarkers as entities that are observed in the body of the human being and that it has not included the measurement process aspect. This exclusion of the measurement aspect was not justified although it is an important aspect of imaging biomarkers (as explained by the QIBA group).

\footnotetext{
2 https://loinc.org/international/

3 http://wwww.who.int/classifications/icd/en/
} 


\subsection{Shortcomings}

All these works are interesting and complementary, but do not provide a solution that is ready to use. The more mature ontology is probably BiomRKRS, but it is not publicly available, and apparently does not cover in detail the domain of imaging biomarkers, in which we are primarily interested. QIBO is also an interesting starting point, although it has many limits. Finally, QIBA protocols constitute interesting contributions, especially concerning protocols (acquisition protocols and reconstruction protocols, image processing) to guarantee the accuracy and reproducibility of the biomarkers. However, such information is not modeled as an ontology but in free text.

\section{Methodology}

Our scope embraces both qualitative and quantitative representations of imaging biomarkers. Indeed, quantitative biomarkers better correspond to what we traditionally consider as a measurement, but it is also important to take into account qualitative measurements which are widely acknowledged in, e.g., radiogenomic studies (Diehn et al., 2008), and therefore interesting to establish correlations between imaging features and tissue pathology at a gene expression level.

\subsection{Alignment to a Foundational Ontology}

Our proposed ontology involves many diverse entities that are related to the imaging biomarker concept, these entities concern many domains (medical imaging, biology, image processing, metrology, clinical research, etc.). In this context, the use of a foundational ontology is important to ensure the semantic consistency of the model. Therefore, IBO relies on BFO (Basic Formal Ontology) as well as on the principles of the OBO (Open Biomedical Ontologies) foundry. Hence, entities are divided into BFO: continuant and BFO:occurrent. The category $\mathrm{BFO}$ : continuant denotes entities that persist through time (medical images, imaging devices, imaging contrast agents, biomarker values, biomarker measurement protocols, etc.) and the category BFO:occurrent represents events in which continuants participate (e.g., imaging biomarker measurement process, imaging biomarker application, etc.). In this work we have reused several ontologies (listed and briefly described hereafter) covering- at least in part-the entities needed in biomarkers modeling. Most of them are aligned with $\mathrm{BFO}$ and were developed according to the OBO foundry principles, thus facilitating the integration work. However in most cases, only subsets of these ontologies are needed.

\subsection{Reuse of Biomedical Ontologies}

In particular, we mainly used the following ontologies to standardize the representation of information that are related to imaging biomarkers:

- Ontology for Biomedical Investigations (OBI) (Brinkman et al., 2010) and Information Artifact Ontology (IAO) (Ceusters, 2012) to represent data related to the measurement process: protocols, used material, generated data (measure, conclusion, predicted value, etc.), study objective, etc.

- Foundational Model of Anatomy (FMA) ontology (Rosse and Mejino J.R., 2003) to represent studied anatomical structure (brain, heart, knee, breast,etc.) of the subject;

- Chemical Entities of Biological Interest (ChEBI) Ontology (Degtyarenko et al., 2008) to specify imaging agents (contrast agents, radiopharmaceuticals, etc.) that are used to help in measuring the imaging parameter;

- Data-set processing (ONL-DP) ontology ${ }^{4}$ to represent original imaging datasets (anatomical, functional, metabolic), image processing processes (registration, re-sampling, segmentation, quantitative parameter estimation, etc.) and processed images (registration data set, segmentation dataset, parameter quantification dataset, etc.).

- Unit ontology (UO) (Gkoutos et al., 2012) to specify the unit of measure of scalar imaging biomarkers.

- Phenotypic Quality Ontology (PATO) (Mungall et al., 2007) to qualify physical characteristics that are measured by imaging biomarkers (e.g., size,shape, structure, radioactivity and concentration).

- Gene Ontology (GO) (Gene Ontology Consortium et al., 2008) to describe biological processes (anisotropic cell growth, cell death, cell division, etc.) that are estimated by imaging biomarkers.

- Human Disease Ontology (HDO) (Schriml, 2016) to refer to the studied pathologies (nervous system disease, cardiovascular system disease, etc.) for which the biomarker is measured.

We adopted a modular architecture, consisting of a main ontology file importing several modules. The main motivation for such a modular architecture was the ability to easily re-extract entities from existing ontologies. However, several situations must be considered,

\footnotetext{
4 http://purl.bioontology.org/ontology/ONL-DP
} 
depending on the reused ontologies. As for BFO2, the whole ontology is imported, since it is a foundational ontology, providing the basic modeling framework for the whole ontology. Concerning the others, e.g. PATO, we selected from PATO the list of classes that seemed to us interesting in the context of imaging biomarkers. We are aware that the decision about the relevant subset is quite subjective, and this is precisely why it is important to have those terms in a module that can be re-extracted if needed in some particular application domain. This strategy is certainly the best possible one with regard to the domain of anatomy, since it is certainly difficult to anticipate all the anatomical terms that may be useful in the imaging biomarkers domain, given the large spectrum of imaging modalities and medical specialties. The solution we provide allows the user to easily re-extract the ontology modules from the original ontology sources, and to tune this extract to their specific application needs.

We used the OntoFox tool ${ }^{5}$ that implements the MIREOT (Minimum Information to Reference an External Ontology Term) methodology (Xiang et al., 2010) to build the modular architecture of IBO that is mainly based on OBO modules from OBI, PATO, IAO, UO, FMA, CHEBI and OGMS. To extract an OBO module from an existing ontology using this tool, three basic parameters must be specified in the input file: source ontology, terms to be extracted (i.e. low level source term URIs, top level source term URIs and target direct superclass URIs and setting for retrieving intermediate source terms) and source annotation URIs. The ontoFox tool automatically generates the extracted OWL module in an RDF/XML format from the input file.

We made our selection of ontologies by taking into account mainly the following aspects: (1) the free availability of the ontology on the web, (2) the good definition of the classes and relationships of the ontology in order to ensure the appropriate reuse of the ontology extracts, (3) the coverage of the targeted domain to create a minimum set of terms to cover our use case and (4) the stability of the ontology, so that future changes do not affect our model. In our work, we analyzed and selected manually classes and relationships to be extracted. The following paragraphs illustrate how we applied these principles in the extraction of OBI and FMA ontologies.

We chose to use OBI for three main reasons. First, $\mathrm{OBI}$ is one of the main ontologies of the OBO library and the most suitable terminological resource that expresses investigations in the oncology field. It has been reused in OBIB (Ontology for Biobanking) (Hewitt and

\footnotetext{
5 http://ontofox.hegroup.org/
}

Watson, 2013; Brochhausen et al., 2016) to semantically describe meta-data of bio-banks (e.g., cancer tumor specimen, genomic data, etc.). Second, OBI meets some of main modeling needs that are expressed in QIBA profiles as the description of entities that describe different types of measurements (scalar value, nominal value, etc.), planned processes (imaging processing, statistical calculation, etc.), imaging devices, roles (patient, participant, study group, etc.), study objectives, experimental protocols and other interesting entities. Added to this, OBI includes annotations, formal descriptions and examples to illustrate the usefulness of entities. Third, in terms of implementation $\mathrm{OBI}$ is easy to integrate given that it is based on the foundational ontology BFO.

Our ontology reuses the following OBI entities: OBI:assay objective, OBI:data transformation objective, OBI:study design, OBI:value specification, OBI:genetic characteristics information, OBI:dose, OBI:study group role, etc. We retained also some subclasses of the entity OBI:planned process like OBI:assay, OBI:data transformation, OBI: investigation, $\mathrm{OBI}$ : investigation, $\mathrm{OBI}$ :material processing, etc.

We chose to use the FMA ontology because it is one of the most "expressive" ontological resources in the biomedical field and a reference ontology for modeling the anatomical structures of the human body. FMA is intended according to its authors to be reused in part and adapted to a specific field. We used the FMA ontology to represent the anatomical sites that are described in radiological reports. We did not use the entire FMA ontology, and we only included key entities that describe anatomical sites of lesions that are cited in (Clunie, 2007). For example, IBO refers to FMA: liver, FMA:pancreas, FMA:breast, FMA:neck, FMA:pelvis, FMA:brain, etc.

\section{Development of the Imaging Biomarker Ontology}

We have designed the IBO ontology in OWL2 (Ontology Web Language) ${ }^{6}$ format using the version 5 of Protégé (Horridge et al., 2014). It contains 4622 concepts, 135 object properties and 3 data properties. The IBO ontology articulates the three basic aspects of imaging biomarkers namely, measured biological characteristic, measurement protocol and role in decision

\footnotetext{
${ }^{6}$ http://www.W3.org/TR/owl2-syntax/
} 
making applications and it is available through this $\operatorname{link}^{7}$.

The set of involved instances in our illustrative examples (see Section 5) has been generated using the Protégé tool. We exploited instances with the CORESE search engine ${ }^{8}$ via the execution of SPARQL queries. Results are presented in an XML format and can be visualized in a structured table via the graphic interface of CORESE.

The following paragraphs introduce the most salient aspects of each of these semantic axes; classes are partitioned into two categories BFO: continuant (see Fig. 1) and BFO: occurrent (see Fig. 2). In this section, labels of terms and relationships are used (rather than actual IRI), for the sake of legibility. The pivotal entity of IBO is IBO:imaging biomarker value, representing the value of the measurement of a biological characteristic, and it results in the realization of a plan specified by a protocol (i.e., IBO:imaging biomarker measurement protocol). An IBO:imaging biomarker value can then be used in various decision processes modeled as IBO: imaging biomarker application.

\subsection{Measurement Process of Imaging Biomarker}

An IBO:imaging biomarker measurement protocol specifies how imaging biomarkers should be produced, as a result of some IBO:imaging biomarker measurement process. Such processes are usually composed of three main sub-processes: IBO: subject preparation (e.g., administration of an imaging agent), $\mathrm{OBI}$ : image creation (the image acquisition process involves physical participation of the subject) and ONL-DP:dataset processing. All these processes are modeled as an OBI:study design execution and associated with the related protocol by the IBO:hasRealizationProtocol object property. The ONL-DP: dataset processing class subsumes these processes: IBO:image reconstruction, ONL-DP:registration, ONL-DP:restoration, ONL-DP:segmentation, IBO: image analysis (leading to biomarker values), etc. The image analysis has two subclasses ONL-DP:quantitative image analysis and IBO:qualitative image analysis.

This part of the ontology plays a major role for specifying how biomarkers should be obtained, and for recording provenance information. This involves many material entities such as QIBO: imaging subject,

\footnotetext{
7 https://medicis.univ-rennes1.fr/_media/members/bernard.gibaud/ibo-final-version.zip?id=members\%3Abernard.gibaud\%3Aindex_cache=cache

8 https://www.w3.org/2001/sw/wiki/Corese
}

OBI:device, QIBO:imaging agent, FMA:anatomical structure, as well as roles realized in the context of the processes in which they participate, e.g., IBO:imaging subject role, OBI:patient role, OBI:material to be added role.

\subsection{Measured Quality of Imaging Biomarker}

An IBO:imaging biomarker value is a subclass of the IAO:measurement datum class that subsumes both categorical and numerical (count, percent, fraction and scalar) values that are defined by the OBI:value specification entity. The qualitative imaging biomarkers include nominal and dichotomous values, nominal and unordered values, and nominal and ordered values. In contrast, quantitative imaging biomarkers define continuous values (ratios, values with units of measure, etc.) and discrete values. Quantitative biomarker values are the output of some ONL-DP:quantitative parameter estimation process, and IBO:qualitative biomarker values are the output of some IBO:qualitative parameter estimation process. Both entities are subsumed by the IBO: image analysis entity.

A key feature of the IBO:imaging biomarker value class is its relationship with the estimated physical characteristic of the imaged object or process. In our work, we have distinguished between two kinds of qualities: qualities that are related to a continuant (e.g., tumor size, tumor volume, etc.) and qualities that describe an occurrent (e.g., the variability of the tumor size value). The relationship between a biomarker value and the quality characterizing the object is modeled by means of the OBI: isQualityMeasurementOf object property. The relationship between a biomarker value and the process being measured is modeled by means of the OBI:isProcessProfileMeasurementOf object property. Both object properties are sub-properties of the IAO: isAbout object property. Qualities of physical objects (e.g., anatomical structures, lesions) are modeled using the PATO:physical object quality entity, whereas process profiles are modeled using BFO:process profile, that we have specialized with the basic process profile categories suggested in the BFO2 documentation, namely IBO:quality process profile, IBO:rate process profile and IBO:beat process profile.

\subsection{Clinical Role of Imaging Biomarker}

IBO recognizes the importance of imaging biomarkers for certain specific clinical purposes, e.g., estab- 


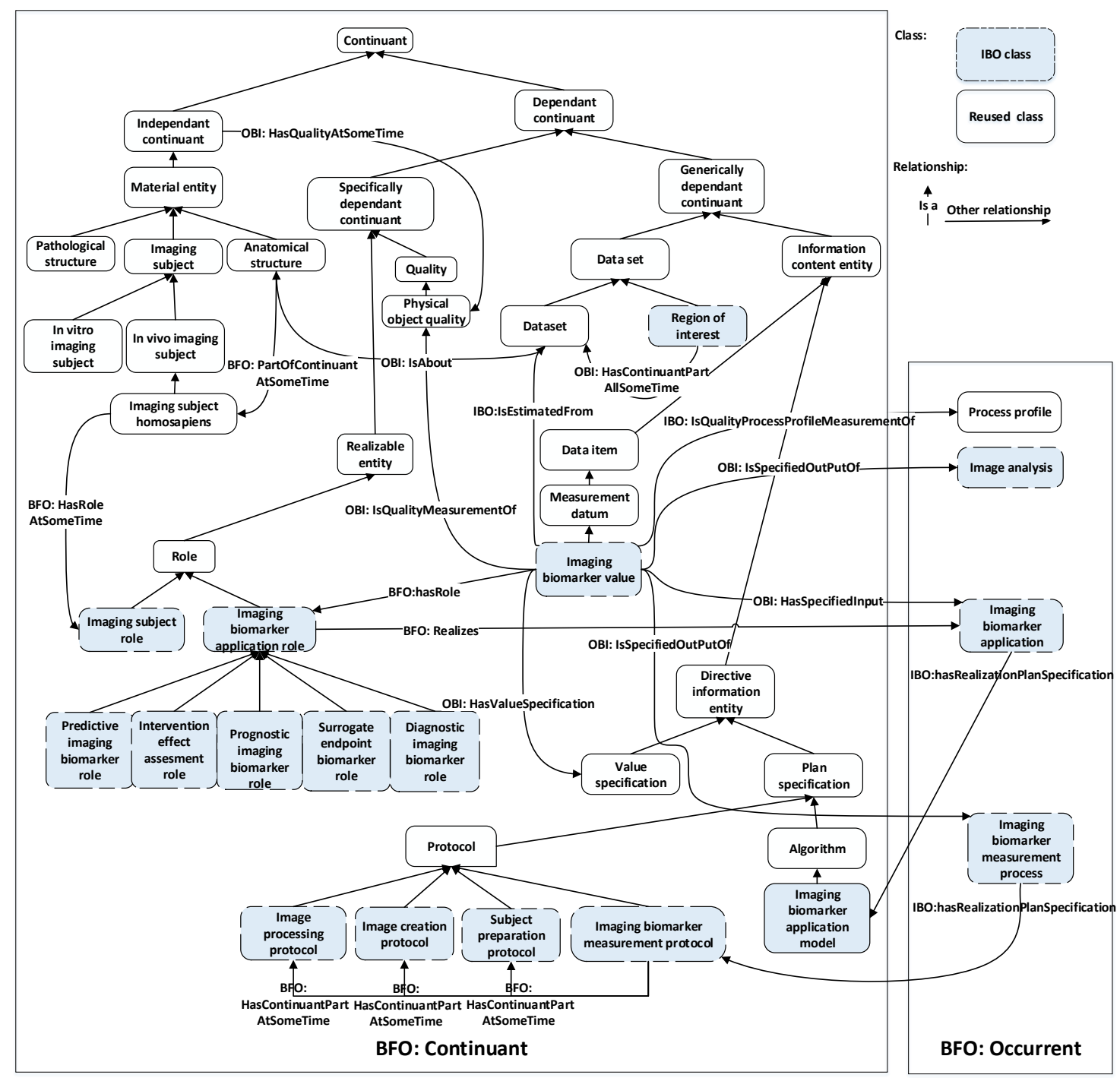

Fig. 1 An extract illustrating the core structure of the BFO:continuant part defining the IBO ontology. Boxes represent classes (blue ones refer to main concepts of semantic axes), and arcs refer to relationships between them: axioms and subsumption relationships. The subsumption "is a" relationship is denoted by unlabeled arcs.

lishing the presence of a disease, predicting the probable outcome of a disease, predicting responses to particular therapies or choose the appropriate drug, monitoring therapy based on the assessment of actual effects. In IBO, all such entities are subsumed by the IBO:imaging biomarker application class that is a subclass of the OBI: data transformation class. An IBO:imaging biomarker application class involves some IBO:imaging biomarker value class which bears a particular role. Each class of biomarker role determines a class of IBO:imaging biomarker application: e.g., IBO:imaging biomarker based diagnosis is an OBI:performing a diagnosis which OBI:hasSpecifiedInput an IBO:image biomarker value that bears some IBO:diagnostic imaging biomarker role. Similar roles were introduced to model prognostic, predictive, effect assessment and surrogate endpoint biomarkers' roles, as illustrated in Fig. 1 .

\section{Application of IBO: TCGA GBM Imaging Features Use Ccase}

In this section, we show how the IBO model can be used to represent imaging features from the Cancer Imaging Archives TCGA (The Cancer Genome Atlas) GBM (glioblastoma multiforme) collection (TCGA-ResearchGroup, 2014). Thus, we consider a retrospective study, 


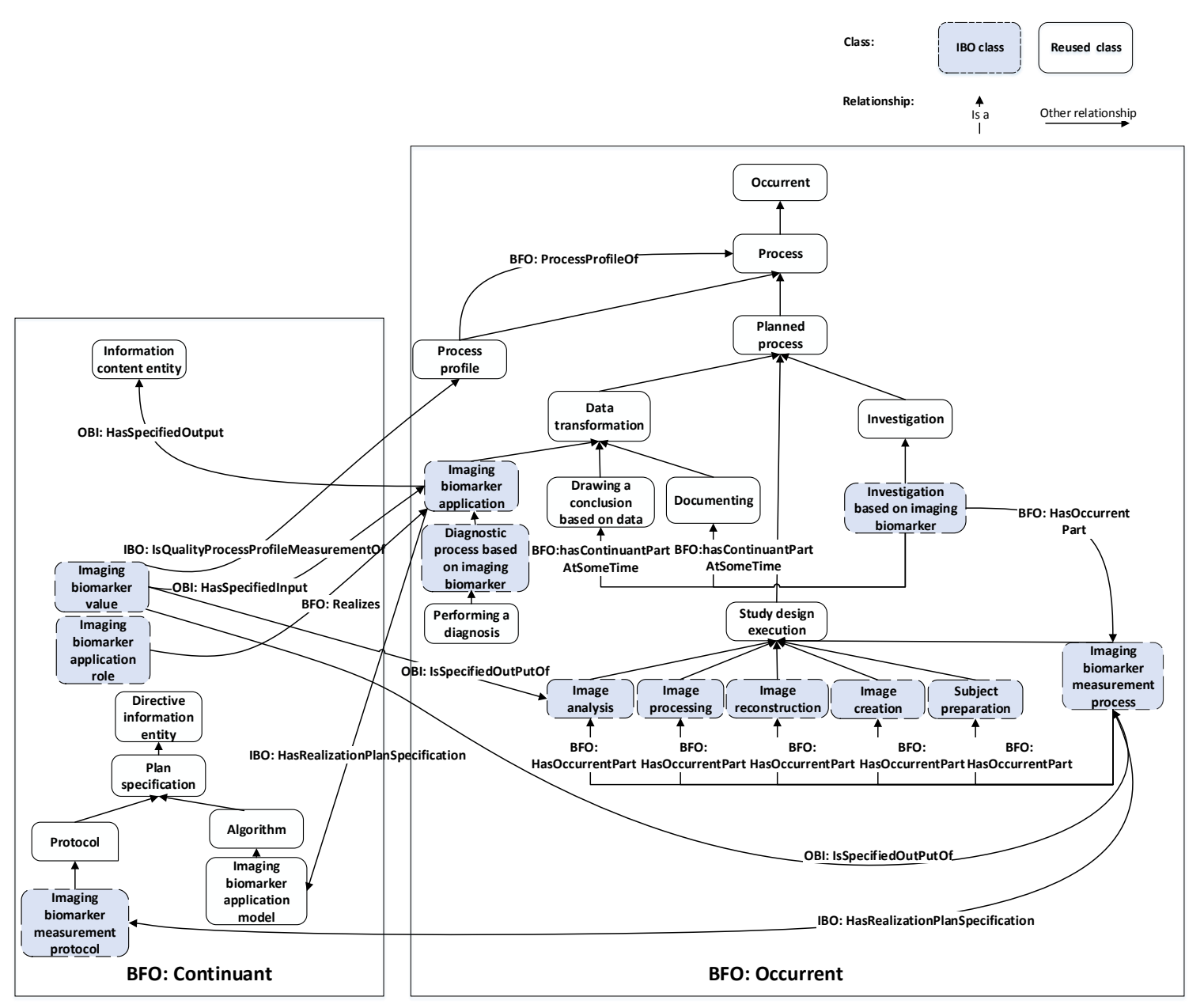

Fig. 2 An extract illustrating the core structure of the BFO:occurrent part defining the IBO ontology. Boxes represent classes (blue ones refer to main concepts of semantic axis), and arcs refer to relationships between them: axioms and subsumption relationships. The subsumption "is a" relationship is denoted by unlabeled arcs.

described in this paper (Jain et al., 2014) that focuses on the combination of morphologic and functional imaging biomarkers of the NER (non-enhancing region) in GBM tumors. The resulted radiology dataset is saved in a spreadsheet file that is available from this $\operatorname{link}^{9}$. This study hypothesizes that morphologic features of the NER are insufficient to predict patient survival and that perfusion parameters namely the relative cerebral blood volume rCBVNER value may lead to a more exact prognostic information.

We note that the Visually Accessible Rembrandt Images terminology called VASARI terminology (TCGA-Research-Group, 2013) was used to describe the non-enhancing part of the tumor. VASARI is a controlled vocabulary that describes thirty observations of gliomas in conventional MRI. For this study, the follow-

\footnotetext{
9 https://wiki.cancerimagingarchive.net/download/attachments/18514300/JainPoisson2014_Radiology_Dataset.xlsx?
}

ing seven VASARI features that describe the NER of the tumor were included: proportion of NER, proportion of edema, definition of NER margins, T1/FLAIR ratio, deep white matter involvement and NER crossing of the midline, and NER area.

The following realistic use-cases illustrate how our model can answer to some competency questions thanks to the axioms that formalize the description of imaging biomarker meta-data.

\subsection{Use case 1: Representation of the Mean rCBV MR} perfusion Parameter

Here, we represent the quantification of the mean rCBV value of the non-enhancing region that is measured from the perfusion imaging. The measuring process includes three main processes namely: subject preparation, image acquisition and image processing. During the prepa- 
ration of the subject, an MRI contrast agent is used to visualize the CER (contrast enhancing region) part. After that, a set of T2 star weighted perfusion images is generated from the image acquisition step. Acquired images are processed to correct contrast agent leakage from the intravascular to the extracellular space, and then, the rCBV value of the CER is estimated from the rCBV map. Fig.3 illustrates the semantic description of the measurement process using the IBO model, and Table 1 specifies some details about the used classes.

Let us consider the query Q1 (see Sect 1.3) to ask for retrieving some meta-data of mean rCBV values (patient, disease, image, image modality, biomarker name, biomarker value) in TCGA studies. Bellow, Q1 is expressed in SPARQL language (Listing 1).

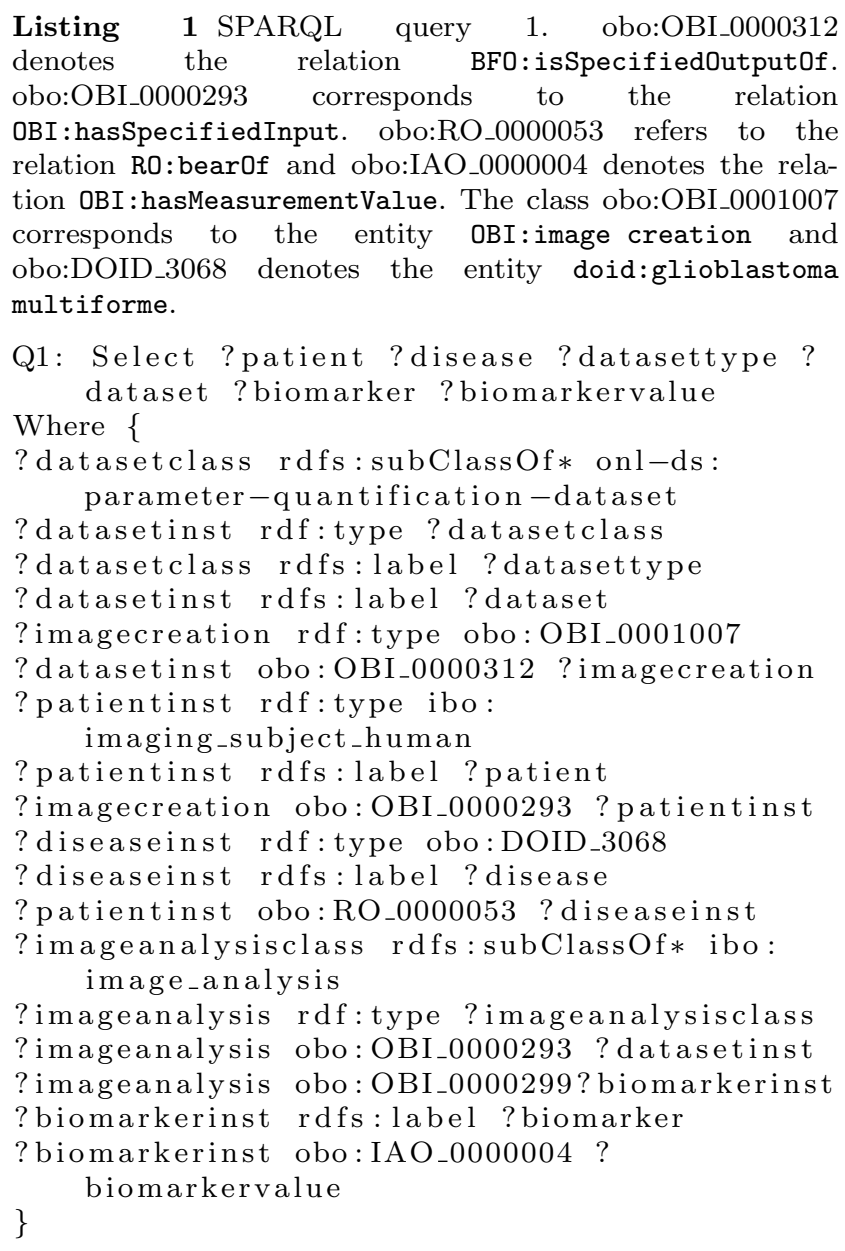

The XML result of the execution of Q1 is generated by CORESE in a table format where the column names are the variables of the SELECT section of the query (Fig. 4).
5.2 Use case 2: Representation of the Non-enhancing Margin Definition

Here, we represent the assessment of the non-enhancing part of the tumor using MR VASARI scores. In this process, radiologists identify the non-enhancing region of the tumor from T1 pre-contrasts and FLAIR MR images. Then, they assign scores to the corresponding VASARI features. In our example, we consider the nonenhancing margin definition criterion that assesses "if most of the outside of the non enhancing margin of the tumor is well defined and smooth versus if the margin is irregular" (TCGA-Research-Group, 2013); the semantic description of this VASARI feature is described in Fig. 5.

In this use case, we consider the competency question Q2 (see Section 1.3) to illustrate how the semantic description of VASARI features can facilitate the retrieval of image content. Listing 2 formulates in SPARQL language Q2 that finds all ROIs of datasets with their associated measures. The result of Q2 is illustrated in figure 6 .

Listing 2 SPARQL query 2. obo:BFO_0000176 denotes the relation $\mathrm{BFO}$ :hasContinuantPartAtSomeTime. obo:OBI_0000312 corresponds to the relation BFO:isSpecifiedOutputOf. obo:IAO_0000004 refers to the relation $\mathrm{OBI}$ :hasMeasurementValue and the class obo:OBI_0000312 denotes the entity OBI:image creation.

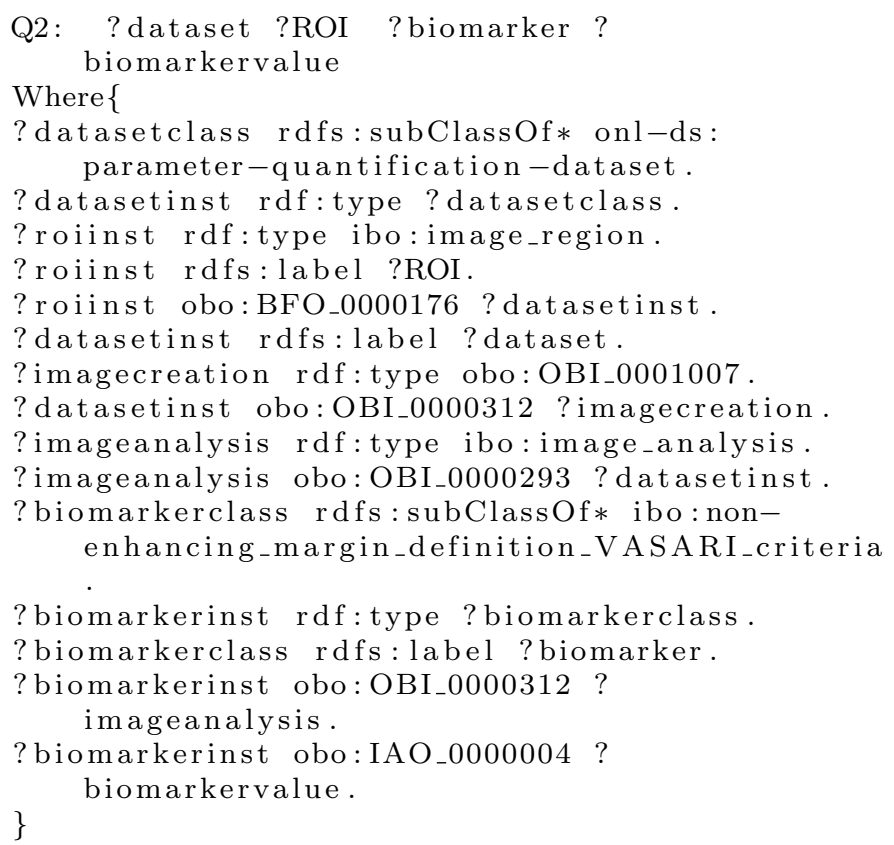

The classification task using the Fact ++ reasoner of Protégé is estimated to $0.25 \mathrm{~s}$. And we should mention that IBO ontology can be used to semantically annotate other GBM imaging biomarkers of the dataset as, for example, the major axis length, the minor axis length and the max of rCBV. 


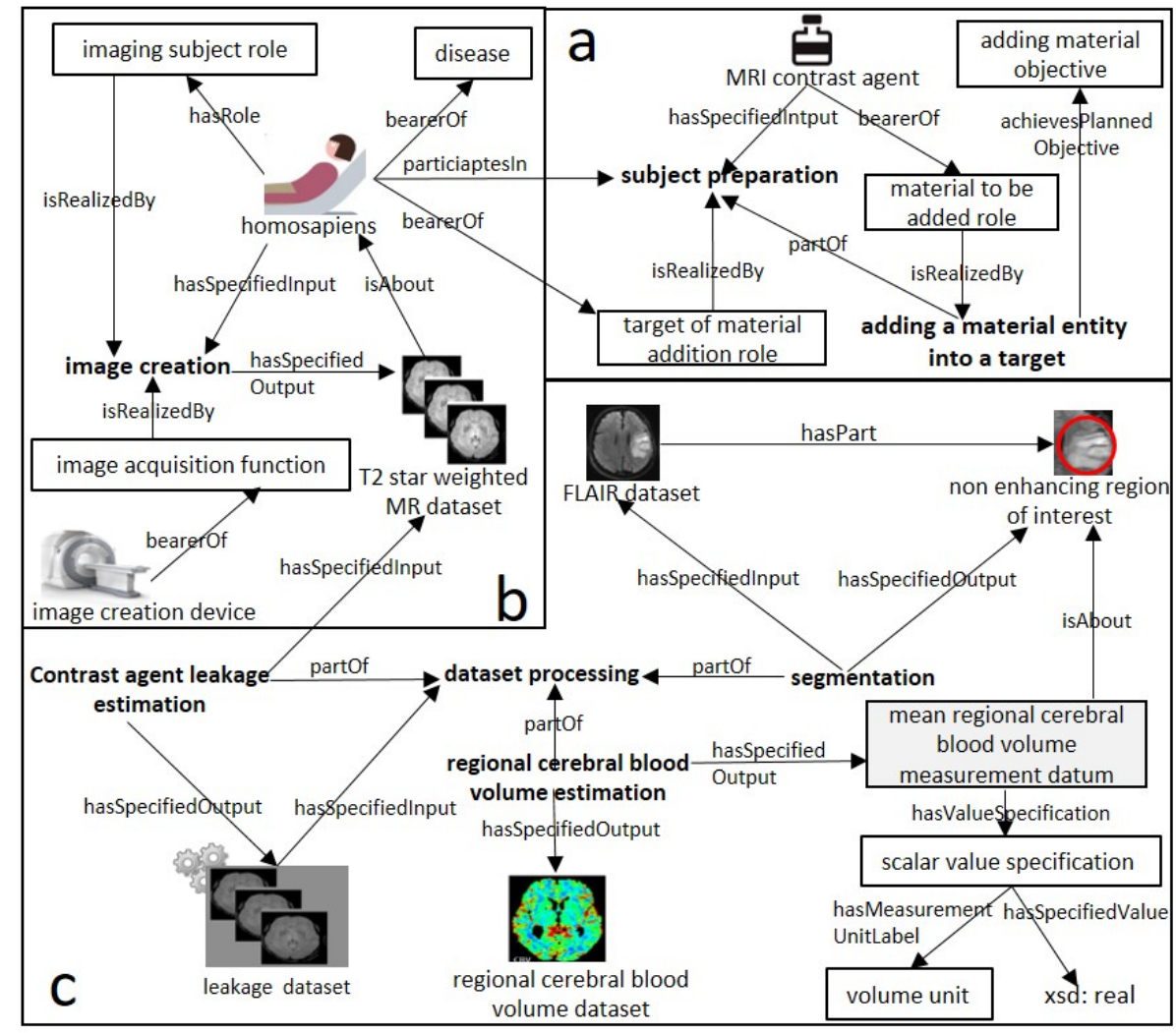

Fig. 3 Representation of the mean rCBV measurement process with the IBO ontology: box a describes the entities that are involved in the subject preparation process, box b corresponds to the image creation process, box c details the dataset processing processes. We note that processes are represented in bold, classes that are not processes are contained in boxes and relationships are denoted with arcs.

\begin{tabular}{|c|c|c|c|c|c|c|c|c|}
\hline Graph & XML & Table & Validate & & & & & \\
\hline \multicolumn{4}{|c|}{ ?patientID } & ?diseasename & ?datas ettype & ?datasetiD & ?biomarkername & ?biomarkervalue \\
\hline \multicolumn{4}{|c|}{ f66d92ff-85ad-4c83-b127-ce34c8488040 } & glioblastoma multiforme & regional cerebral blood volume dataset & TCGA-06-0127-rcbvd & mean rcbv & 4,243 \\
\hline \multicolumn{4}{|c|}{$31 \mathrm{e} 6 \mathrm{e} 6 \mathrm{c} 8-9197-4927-\mathrm{bfe} 4-8 \mathrm{f} 6836 \mathrm{f} 963 \ldots$} & glioblastoma multiforme & regional cerebral blood volume dataset & TCGA-06-0128-rcbvd & mean rcbv & 1,388 \\
\hline \multicolumn{4}{|c|}{ 9ad6c241-ccc5-4532-a6ac-098271b13. } & glioblastoma multiforme & regional cerebral blood volume dataset & TCGA-06-0132-rCbvd & mean rcbv & 2,173 \\
\hline \multicolumn{4}{|c|}{$0133 \mathrm{e} 584-111 \mathrm{e}-450 \mathrm{a}-\mathrm{b} 451-77$ a2799ef } & glioblastoma multiforme & regional cerebral blood volume dataset & TCGA-06-0133-rcbvd & mean rcbv & 2,435 \\
\hline \multicolumn{4}{|c|}{ d0de6676-6ba1-4d79-a9b0-ec3f1e8a8 } & glioblastoma multiforme & regional cerebral blood volume dataset & TCGA-06-0137-rcbvd & mean rcbv & 2,53 \\
\hline \multicolumn{4}{|c|}{ 5bbeb8a7-3ac2-4ef1-bd48-6f83bc994a. } & glioblastoma multiforme & regional cerebral blood volume dataset & TCGA-06-0139-rcbvd & mean rcbv & 2,112 \\
\hline \multicolumn{4}{|c|}{ 357e2 b63-6876-4c69-9728-c52fada81.. } & glioblastoma multiforme & regional cerebral blood volume dataset & TCGA-06-0143-rcbvd & mean rcbv & 2,393 \\
\hline \multicolumn{4}{|c|}{ 562d2831-ea9e-4ee1-bcee-77138e0a... } & glioblastoma multiforme & regional cerebral blood volume dataset & TCGA-06-0147-rcbvd & mean rcbv & 2,24 \\
\hline \multicolumn{4}{|c|}{$9 a d 6535 c$-da13-4f16-9df9-2b5df9aafe $8 c$} & glioblastoma multiforme & regional cerebral blood volume dataset & TCGA-06-0164-rcbvd & mean rcbv & 1,768 \\
\hline \multicolumn{4}{|c|}{$4 a 85 a 43 b-4 d b c-4081-b 552-d f 06 e e f 1 a \ldots$} & glioblastoma multiforme & regional cerebral blood volume dataset & TCGA-06-0166-rcbvd & mean rcbv & 3,038 \\
\hline \multicolumn{4}{|c|}{$224235 c 1-5 b 6 e-48 d 5-a 5 d 1-777 d f e d e 0}$. & glioblastoma multiforme & regional cerebral blood volume dataset & TCGA-06-0168-rcbvd & mean rcbv & 1,37 \\
\hline \multicolumn{4}{|c|}{ ada9c707-d49c-43d5-bee6-3e007327 } & glioblastoma multiforme & regional cerebral blood volume dataset & TCGA-06-0173-rcbvd & mean rcbv & 2,027 \\
\hline \multicolumn{4}{|c|}{ be8fa1fb-5696-4719-af16-2059f6b9d270 } & glioblastoma multiforme & regional cerebral blood volume dataset & TCGA-06-0175-rcbvd & mean rcbv & 2,12 \\
\hline \multicolumn{4}{|c|}{ bdb95e64-da10-4f2a-bac9-9a534cf564 } & glioblastoma multiforme & regional cerebral blood volume dataset & TCGA-06-0177-rcbvd & mean rcbv & 1,368 \\
\hline \multicolumn{4}{|c|}{ f3158197-173b-4917-ac3b-ed3ba04a5... } & glioblastoma multiforme & regional cerebral blood volume dataset & TCGA-06-0179-rcbvd & mean rcbv & 2,51 \\
\hline
\end{tabular}

Fig. 4 Table displaying the results of Q1 query in CORESE

These two use cases illustrate that the use of OWL for the description of imaging results allows their formal processing. Thus for example, the annotation of imaging biomarkers with IBO should mainly allow: the consistent description of the input and output data, the aggregation of disperse imaging datasets, the performance of advanced search capabilities in clinical or research contexts, etc. Thanks to the knowledge embed- ded in the ontology, IBO can respond to diverse relevant queries.

The annotation of imaging result data with the same ontology can insure a sort of a collaboration between multiple investigators creating data about entities of the same types. Thus, data providers will not organize the data based on their predefined schema but, they will adopt a federated approach in data sharing. 
Table 1 Used classes in the use cases 1 and 2

\begin{tabular}{|c|c|}
\hline Class label & Parent class label \\
\hline IBO: subject preparation & OBI:planned process \\
\hline OGMS: disease & BFO:disposition \\
\hline OBI:target to material addition role & BFO:role \\
\hline OBI:material to be added role & BFO:role \\
\hline OBI:MRI contrast agent & CHEBI : pharmaceutical \\
\hline $\begin{array}{l}\text { OBI :adding a material entity into a } \\
\text { target }\end{array}$ & OBI:material combination \\
\hline OBI: adding material objective & OBI:material combination objective \\
\hline OBI: image creation & OBI:planned process \\
\hline OBI: homosapiens & $\mathrm{OBI}$ : organism \\
\hline IBO: imaging subject role & BFO:role \\
\hline ONL-DP:T2 star weighted MR dat & $\begin{array}{l}\text { ONL-DP: functional dataset, } \quad \text { ONL-DP:MR } \\
\text { dataset, ONL-DP:reconstructed-dataset }\end{array}$ \\
\hline OBI:image acquisition function & OBI:measure function \\
\hline OBI: image creation device & OBI: device \\
\hline ONL-DP: dataset processing & OBI:data transformation \\
\hline ONL-DP: segmentation & ONL-DP: dataset processing \\
\hline IBO:non enhancing region of interest & ONL-DP: segmentation dataset \\
\hline IBO:FLAIR dataset & ONL-DP: parameter quantification dataset \\
\hline $\begin{array}{l}\text { ONL-DP:regional cerebral blood volume } \\
\text { estimation }\end{array}$ & ONL-DP:quantitative parameter estimation \\
\hline $\begin{array}{l}\text { ONL-DP:regional cerebral blood volume } \\
\text { dataset }\end{array}$ & $\begin{array}{l}\text { ONL-DP: parameter quantification dataset, } \\
\text { ONL-DP: hemodynamic dataset }\end{array}$ \\
\hline $\begin{array}{l}\text { IBO:mean regional cerebral blood volume } \\
\text { measurement datum }\end{array}$ & $\begin{array}{l}\text { OBI: average value, IBO:imaging biomarker } \\
\text { volume measurement datum }\end{array}$ \\
\hline OBI:scalar value specification & OBI:numeric value specification \\
\hline UD: volume unit & IAD:measurement unit label \\
\hline IBO:qualitative parameter estimation & IBO:parameter estimation \\
\hline IBO:non enhancing margin definition & IBO:categorical VASARI criterion \\
\hline $\begin{array}{l}\text { IBO: non enhancing margin definition } \\
\text { value specification }\end{array}$ & IBO:categorical value specification \\
\hline $\begin{array}{l}\text { IBO: non enhancing margin definition } \\
\text { label option }\end{array}$ & IBO: categorical label \\
\hline
\end{tabular}

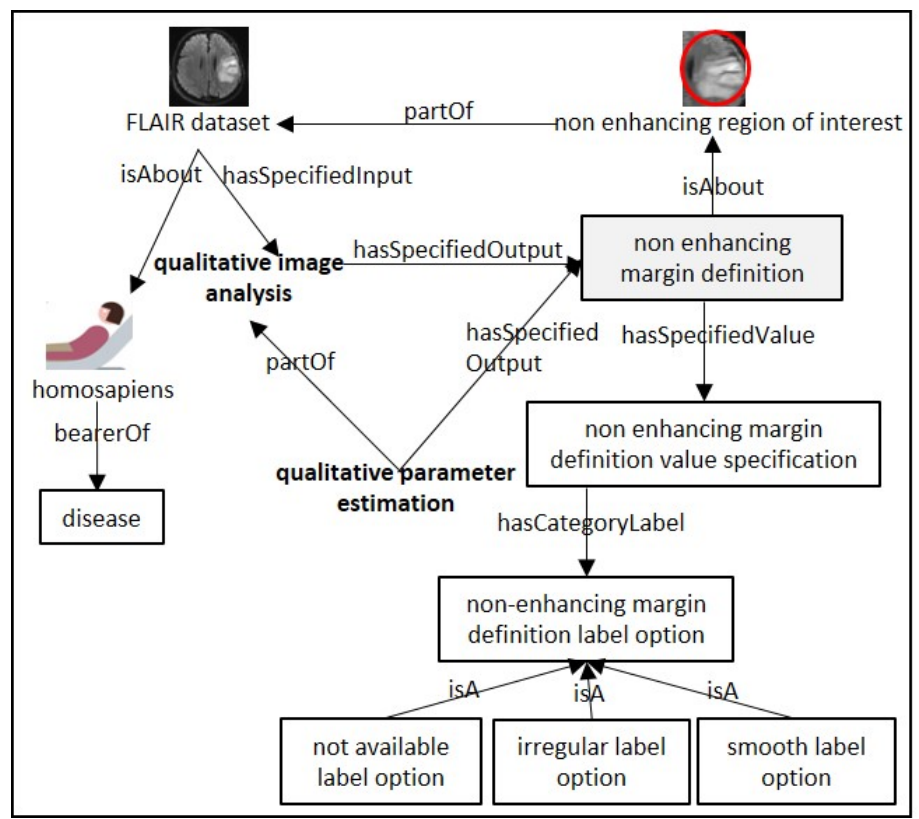

Fig. 5 Representation of the VASARI feature non-enhancing margin definition with the IBO ontology. We note that processes are represented in bold, classes that are not processes are contained in boxes and relationships are denoted with arcs 


\begin{tabular}{|c|c|c|c|c|c|}
\hline Graph & XML & Table & Validate & & \\
\hline \multicolumn{3}{|c|}{ ?dataset } & ?ROI & ?biomarker & ?biomarkervalue \\
\hline \multicolumn{3}{|c|}{ TCGA-06-0127-T2-star } & TCGA-06-0127-ner & non-enhancing margin definition VASARI criteria & Poorly-defined \\
\hline \multicolumn{3}{|c|}{ TCGA-06-0128-T2-star } & TCGA-06-0128-ner & non-enhancing margin definition VASARI criteria & well defined \\
\hline \multicolumn{3}{|c|}{ TCGA-06-0132-T2-star } & TCGA-06-0132-ner & non-enhancing margin definition VASARI criteria & well defined \\
\hline \multicolumn{3}{|c|}{ TCGA-06-0133-T2-star } & TCGA-06-0133-ner & non-enhancing margin definition VASARI criteria & well defined \\
\hline \multicolumn{3}{|c|}{ TCGA-06-0137-T2-star } & TCGA-06-0137-ner & non-enhancing margin definition VASARI criteria & well defined \\
\hline \multicolumn{3}{|c|}{ TCGA-06-0139-T2-star } & TCGA-06-0139-ner & non-enhancing margin definition VASARI criteria & poorly defined \\
\hline \multicolumn{3}{|c|}{ TCGA-06-0143-T2-star } & TCGA-06-0143-ner & non-enhancing margin definition VASARI criteria & well defined \\
\hline \multicolumn{3}{|c|}{ TCGA-06-0147-T2-star } & TCGA-06-0147-ner & non-enhancing margin definition VASARI criteria & well defined \\
\hline \multicolumn{3}{|c|}{ TCGA-06-0149-T2-star } & TCGA-06-0149-ner & non-enhancing margin definition VASARI criteria & poorly defined \\
\hline \multicolumn{3}{|c|}{ TCGA-06-0149-T2-star } & TCGA-06-0149-ner & non-enhancing margin definition VASARI criteria & well defined \\
\hline \multicolumn{3}{|c|}{ TCGA-06-0166-T2-star } & TCGA-06-0166-ner & non-enhancing margin definition VASARI criteria & well defined \\
\hline \multicolumn{3}{|c|}{ TCGA-06-0168-T2-star } & TCGA-06-0168-ner & non-enhancing margin definition VASARI criteria & poorly defined \\
\hline \multicolumn{3}{|c|}{ TCGA-06-0173-T2-star } & TCGA-06-0173-ner & non-enhancing margin definition VASARI criteria & poorly defined \\
\hline \multirow{2}{*}{\multicolumn{3}{|c|}{$\begin{array}{l}\text { TCGA-06-0177-T2-star } \\
\text { TCGA-06-0179-T2-star }\end{array}$}} & TCGA-06-0177-ner & non-enhancing margin definition VASARI criteria & well defined \\
\hline & & & TCGA-06-0179-ner & non-enhancing margin definition VASARI criteria & well defined \\
\hline
\end{tabular}

Fig. 6 Table displaying the results of query Q2 in CORESE

Table 2 Top-level classes mapping to cover the upper-level classes of the QIBO ontology.

\begin{tabular}{lll}
\hline Classes QIBO & Correspondence & IBO representation \\
\hline QIBO:biological target & $\subseteq$ & OBI : target of material addition \\
QIBO:biological intervention & $=$ & OBI:material processing \\
QIBO:biomarker use & $\subseteq$ & OBI:planned process \\
QIBO: imaging agent & $\subseteq$ & OBI:material to be added \\
QIBO:imaging subject & $\subseteq$ & IBO: imaging subject role \\
QIBO:imaging technique & $=$ & ONL-DP:dataset \\
QIBO:indicated biology & $\subseteq$ & GO:biological process, OGMS:disease \\
QIBO: post-processing algorithm & $\subseteq$ & OBI:data transformation \\
QIBO:quantitative imaging biomarker & $\subseteq$ & OBI:measurement datum \\
\hline
\end{tabular}

We note that the symbol $=$ denotes that the two referred classes are equivalent and the symbol $\subseteq$ denotes a subsumption relation

\section{Discussion}

Our work shows that it is possible to cover the main three semantic axes of the imaging biomarker concept by integrating and specializing classes from existing biomedical ontologies. We have created a generic ontology whose main objective is to define precisely this concept of imaging biomarker and to remove the ambiguity regarding it. Throughout our work, we have taken into consideration the limits of previous stateof-the art works. We have followed an ontology development methodology that is different from QIBO; three major differences can be mentioned. First, we created IBO using concepts coming from specialized ontologies (FMA, OBI, PATO, etc.) and that are well recognized by the OBO community, and others such as ONL-DP focusing medical imaging data. Second, IBO has been built in a modular way that facilitates its reuse and potential extension by future users. (Subsets of external ontologies are extracted with the OntoFox tool.) finally, unlike QIBO, which was not aligned with a high-level ontology, IBO is based on BFO. (Table 2 shows how QIBO main semantic axes are represented in the IBO ontology with extended or new terms from terms other specialized ontologies.)
We have modeled the concept of imaging biomarker in a different way from that proposed in (Ceusters and Smith, 2015). Contrary to their proposal, our proposal articulates the concept of biomarker with the aspects of protocol and measurement process. We made this choice to take into account the basic aspect of the imaging biomarker concept that a biomarker is a characteristic that is objectively evaluated and measured. According to the Institute of Medicine and to the QIBA group, the term objectively means precisely and reproducibly. However, these two aspects of accuracy and reproducibility can only be achieved by defining measurement protocols. This approach differs from that of Ceusters and Smith (2015), for whom objectivity is related to the intrinsic properties of observed quality and not to the measurement process.

We believe that the concept of imaging biomarker is better represented with IBO for the two following reasons. First, we have added fundamental concepts that are not present in QIBO, BiomRKRS and the work of Ceusters et al., such as qualitative biomarkers, regions of interest, measurement protocols and the roles of imaging biomarkers. Whereas Ceusters and Smith (2015) introduce biomarkers as a disjunction of the three categories of imaging biomarkers, we have articulated the latter explicitly. The main limitation of our contribution is that IBO must be extended by other specific classes before its application to a specific pathology. (QIBO and BiomRKRS have the same 
limitation.) In this paper, we have demonstrated how IBO can be used to annotate important imaging features in the glioblastoma domain and for these two use cases we extended our ontology to cover some specific needs of the GBM domain. But unfortunately, to cover other use cases it will be necessary to adapt the model to some specific needs of medical application.

\section{Conclusion}

The importance of imaging biomarkers in biomedical research and drug design is well acknowledged in the literature, calling for appropriate standards and guidelines for biomarker development, validation and qualification. Beyond that, the development of precision medicine, the key role that imaging biomarkers will play in medical decision processes and the development of decision support systems make it absolutely necessary to define explicit and consensual semantics of the conceptual entities within this complex domain. The IBO core ontology of imaging biomarkers is a first step in this direction that reuses preliminary work from QIBO and BiomRKRS as well as relevant biomedical ontologies.

\section{References}

R.G. Abramson and T.E. Yankeelov. Imaging biomarkers and surrogate endpoints in oncology clinical trials. In Functional imaging in oncology, pages 29-42. Springer, 2014.

M. Asslaber and K. Zatloukal. Biobanks: transnational, european and global networks. Brief Funct Genom Proteom, 6(3):193-201, 2007.

J.R. Ball, C.M. Micheel, et al. Evaluation of biomarkers and surrogate endpoints in chronic disease. National Academies Press, Washington, 2010.

J.M.L. Bosmans, L. Peremans, M. Menni, A.M. De Schepper, P.O. Duyck, and P.M. Parizel. Structured reporting: if, why, when, how and at what expense? results of a focus group meeting of radiology professionals from eight countries. Insights Imaging, 3(3):295-302, 2012.

R.R. Brinkman, M. Courtot, D. Derom, J. Fostel, Y. He, P.W. Lord, J. Malone, H.E. Parkinson, B. Peters, P. Rocca-Serra, et al. Modeling biomedical experimental processes with OBI. J. Biomed Semant, $1(\mathrm{~S}-1): \mathrm{S} 7,2010$.

M. Brochhausen, J. Zheng, D. Birtwell, H. Williams, A.M. Masci, H.J. Ellis, and C.J. Stoeckert. Obib-a novel ontology for biobanking. J Biomedical semant, $7(1): 23,2016$.
M. Brunnbauer. Dicom metadata as rdf. In GIJahrestagung, pages pp 1796-1804, 2013.

A.J. Buckler, L. Bresolin, N.R. Dunnick, and D.C. Sullivan. A collaborative enterprise for multi-stakeholder participation in the advancement of quantitative imaging. Radiology, 258(3):906-914, 2011.

A.J. Buckler, M. Ouellette, J. Danagoulian, G. Wernsing, E. Savig, Savig E Liu TT, B.E. Suzek, and Rubin DL Paik D. Quantitative imaging biomarker ontology (QIBO) for knowledge representation of biomedical imaging biomarkers. J Digit Imaging, 26 (4):630-641, 2013.

W. Ceusters. An information artifact ontology perspective on data collections and associated representational artifacts. In MIE, pages pp 68-72, 2012.

W. Ceusters and B. Smith. Biomarkers in the ontology for general medical science. In Cornet $R(e d$.$) , Digital$ healthcare empowering Europeans, Vol 512(no 8):pp 155-159, 2015.

D.S. Channin, P. Mongkolwat, V. Kleper, K. Sepukar, and D.L. Rubin. The caBIG annotation and image markup project. J Digit Imaging, 23(2):217-225, 2010.

D.A. Clunie. DICOM structured reporting. PixelMed Publishing, Bangor, 2000.

D.A. Clunie. Encoding of oncology clinical trial read results in DICOM SR. http://qibawiki.rsna.org/images/f/fc/ ClinicalTrialResults_20111123_QIBA.doc, 2007.

K. Degtyarenko, P. De Matos, M. Ennis, J. Hastings, M. Zbinden, A. McNaught, R. Alcántara, M. Darsow, M.l Guedj, and M. Ashburner. ChEBI: a database and ontology for chemical entities of biological interest. Nucl Acids Res, 36(suppl 1):D344-D350, 2008.

M. Diehn, C. Nardini, D.S. Wang, S. McGovern, M. Jayaraman, Y.U. Liang, K. Aldape, S. Cha, and M.D. Kuo. Identification of noninvasive imaging surrogates for brain tumor gene-expression modules. Proc Natl Acad Sci, 105(13):5213-5218, 2008.

E.A. Eisenhauer, Bogaerts J. Therasse, P., L.H. Schwartz, D. Sargent, R. Ford, J. Dancey, S. Arbuck, S. Gwyther, Mooney M., et al. New response evaluation criteria in solid tumours: revised RECIST guideline (version 1.1). Eur J Cancer, 45(2):228-247, 2009.

ESR. White paper on imaging biomarkers. Insights Imaging, 1(2):42-45, 2010.

Gene Ontology Consortium et al. The gene ontology project in 2008. Nucl Acids Res, 36(suppl 1):D440D444, 2008.

G.V. Gkoutos, P.N. Schofield, and R. Hoehndorf. The units ontology: a tool for integrating units of measurement in science. https://doi.org/10.1093/ 
database/bas033, 2012.

R. Hewitt and P. Watson. Defining biobank. Biopreserv and biobank, 11(5):309-315, 2013.

M. Horridge, T. Tudorache, C. Nuylas, J. Vendetti, N.F. Noy, and M.A. Musen. Webprotégé: a collaborative web-based platform for editing biomedical ontologies. Bioinformatics, 30(16):2384-2385, 2014.

F.A. Jaffer and R. Weissleder. Molecular imaging in the clinical arena. Jama, 293(7):855-862, 2005.

R. Jain, L.M. Poisson, D. Gutman, L. Scarpace, S.N. Hwang, C.A. Holder, M. Wintermark, A. Rao, R.R. Colen, J. Kirby, et al. Outcome prediction in patients with glioblastoma by using imaging, clinical, and genomic biomarkers: focus on the nonenhancing component of the tumor. Radiology, 272(2):484-493, 2014.

G.J. Kelloff, R.C. Bast, D.S. Coffey, A.V. DAmico, R.S. Kerbel, J.W Park, R.W. Ruddon, G.J.S. Rustin, R.L. Schilsky, C.C. Sigman, et al. Biomarkers, surrogate endpoints, and the acceleration of drug development for cancer prevention and treatment an update prologue. Clin Cancer Res, 10(11):3881-3884, 2004.

L.G. Kessler, H.X. Barnhart, A.J. Buckler, K.R. Choudhury, M.V. Kondratovich, A. Toledano, A.R. Guimaraes, R. Filice, Z. Zhang, and D.C. Sullivan. The emerging science of quantitative imaging biomarkers terminology and definitions for scientific studies and regulatory submissions. Stat Methods Med Res, 24(1):9-26, 2015.

J. Malone, E. Holloway, T. Adamusiak, M. Kapushesky, J. Zheng, N. Kolesnikov, A. Zhukova, A. Brazma, and H. Parkinson. Modeling sample variables with an experimental factor ontology. Bioinformatics, 26 (8):1112-1118, 2010.

C. Mungall, G. Gkoutos, N. Washington, and S. Lewis. Representing phenotypes in OWL. In In Golbreich $C$, Kalyanpur $A$, Parsia $B$ (eds) Proceedings of the OWLED 2007 workshop on OWL: experience and directions. Innsbruck, Austria, 2007.

J.P.B. O'Connor, A. Jackson, M.C. Asselin, D.L. Buckley, G.J.M. Parker, and G.C. Jayson. Quantitative imaging biomarkers in the clinical development of targeted therapeutics: current and future perspectives. Lancet Oncol, 9(8):766-776, 2008.

B. Ofoghi, G.H.L. Campos, K. Verspoor, and F.J.M. Sanchez. BiomRKRS: a biomarker retrieval and knowledge reasoning system. In Proceedings of the seventh Australasian workshop on health informatics and knowledge management-Vol 153, pages 31-39. Australian Computer Society, Inc., 2014.

C. Rosse and José L.V. Mejino J.R. A reference ontology for biomedical informatics: the foundational model of anatomy. J Biomedical Inform, 36(6):478-
500, 2003.

RSNA. Quantitative imaging biomarkers alliance. https://www.rsna.org/QIBA.aspx, [online] accessed 18 july 2018, 2007.

D.L. Rubin, C. Rodriguez, P. Shah, and C.F. Beaulieu. iPad: Semantic annotation and markup of radiological images. In $A M I A, 2008$.

R.H. Scheuermann, W. Ceusters, and B. Smith. Toward an ontological treatment of disease and diagnosis. Transl Bioinform, 2009:116, 2009.

L. Schriml. Human disease ontology. 2016.

TCGA-Research-Group. Wiki for the Vasari feature set. Vasari research project. https: //wiki.cancerimagingarchive.net/display/ Public/VASARI+Research+Project, 2013.

TCGA-Research-Group. The Cancer Genome Atlas glioblastoma multiforme data collection. https://wiki.cancerimagingarchive.net/ display/Public/TCGA-GBM, 2014.

J.A. Wagner, S.A. Williams, and C.J. Webster. Biomarkers and surrogate end points for fit-forpurpose development and regulatory evaluation of new drugs. Clin Pharmacol Ther, 81(1):104-107, 2007.

Z. Xiang, M. Courtot, R.R. Brinkman, A. Ruttenberg, and Y. He. Ontofox: web-based support for ontology reuse. BMC Res Notes, 3(1):175, 2010. 